\section{Opioid abuse in children: an emerging public health crisis in the United States!}

Advance online publication, 2 August 2017; doi:10.1038/pr.2017.166

To the Editor: Opioid abuse by adults is widely perceived as a major health hazard in the United States of America $(1,2)$. From 1997 to 2012 hospitalizations attributed to opioid poisonings in children raised by nearly twofold (3). Further, Emergency Department (ED) visits by young children and adolescents

for poisoning by prescription opioids is well described (4). However, current national estimates of opioid abuse by children who visit the ED for any cause are relatively unknown. Understanding and characterizing opioid abuse in children at a national level is the initial step in optimizing the overall care for children. In this initial exploratory study, we sought to estimate the burden of opioid dependence/abuse $(\mathrm{OD} / \mathrm{OA})$ in children who visited the ED for any cause in the United States. We hypothesize that there is an increasing trend of $\mathrm{OD} / \mathrm{OA}$ in children who visited the $\mathrm{ED}$ during the study period.

We performed a retrospective analysis of the Nationwide Emergency Department Sample (NEDS) (5) for the years 20082013. The NEDS is the largest all-payer ED database in the United States. Discharge data for ED visits from nearly 945 hospitals located across major states in the USA, approximating a $20 \%$ stratified sample of US hospital-based EDs are available in NEDS. Weighted, NEDS contains estimates of roughly 135 million ED visits. All ED visits with ICD-9-CM diagnoses codes for OD/OA (304.00-03; 305.50-53) among those aged up to 21 years were selected. Demographic characteristics of this cohort were examined. Multivaraiable linear and logistic regression models were used to examine ED charges and disposition status following the ED visit.

During the study period, a total of 257,165 ED visits with $\mathrm{OD} / \mathrm{OA}$ among those aged up to 21 years were made in the United States. The number of ED visits increased from 32,235 in year 2008 to 49,626 in year 2013 (Figure 1). In all, 88.3\% of ED visits were made by those aged $18-21$ years and $8.4 \%$ of ED visits were made by those aged 16-17 years. Males comprised $56 \%$ of ED visits. Types of OD included: unspecified $(N=79,787)$, continued $(N=44,960)$, episodic $(N=723)$, and in remission $(N=3,948)$. Types of $\mathrm{OA}$ included: unspecified $(N=111,219)$, continued $(N=11,182)$, episodic $(N=1,841)$, and in remission $(N=4,913)$. Following an $\mathrm{ED}$ visit, $58.2 \%$ were discharged routinely and $31.4 \%$ were admitted as inpatients in the same hospital as the ED visit.
All-cause mortality: 200 children died in the ED, whereas 325 died following hospitalization. Predominant primary payers included Medicaid (27.1\% of ED visits) and private insurance (41.4\%); $24.8 \%$ were uninsured. Close to $92 \%$ of patients did not have any chronic co-morbid condition (CMC). The mean ED charge per visit was $\$ 2,372$ and the total ED charge across the entire USA during the study period was $\$ 505$ million (USD value as in the year 2013). The mean hospitalization charge was $\$ 25,356$ and total hospitalization charge across the entire USA was $\$ 2$ billion. Those with significantly higher ED charges included those diagnosed with an unspecified OA $(P<0.01)$, those aged $13-15$ years $(P=0.04)$, those aged 16-17 years $(P=0.01)$, females $(P=0.04)$, those with CMC $(P=0.03)$, and those residing in western regions of USA $(P<0.01)$. Those aged up to 9 years $(P<0.01)$, those with an increasing co-morbid burden $(P<0.01)$, those residing in the northeast/midwest/southern regions $(P<0.01)$, and those residing in high-income households $(P<0.01)$ were more likely to be hospitalized as opposed to being routinely discharged from the ED. The uninsured were less likely to be hospitalized $(P<0.01)$.

Limitations of the present study include the use of administrative data set with the potential for underestimating the actual problem. Nevertheless, the utility of NEDS for exploratory studies is well established (3-5). Clear guidelines are lacking pertaining to the testing of $\mathrm{OA}$ in children in various medical settings. In this initial exploratory study, the uninsured children with OA/OD were less likely to be hospitalized compared to their counterparts. In our opinion this is an important "signal" that merits further research. In the present study, we sought to estimate the burden of $\mathrm{OA} / \mathrm{OD}$ in children who visited the ED for "any" cause. The causes of ED visits are a very heterogeneous group of diagnosis in this population. Adjustment for all diagnosis to seek an association between insurance status and $\mathrm{OA} / \mathrm{OD}$ hospitalization is beyond the scope of the present study. In addition, the severity of the chief complaint (and the primary diagnosis) could influence the decision to hospitalize the patient irrespective of OA/OD. Nevertheless, this is an interesting finding that merits closer evaluation. The results of this study are exploratory and we hope will kindle the interest of the public, the researchers, and the administrators to further define and address this important public health problem in children in the United States.

In conclusion, opioid abuse is a major public health issue in children in the United States. Nearly, 117 children test positive for OA/OD every day across the EDs in the United States. There is an increasing trend in the use of opioids by children. Common characteristics are described in this exploratory study. Further studies are needed to identify OA in other medical settings as well! 


\section{Letter to the Editor}

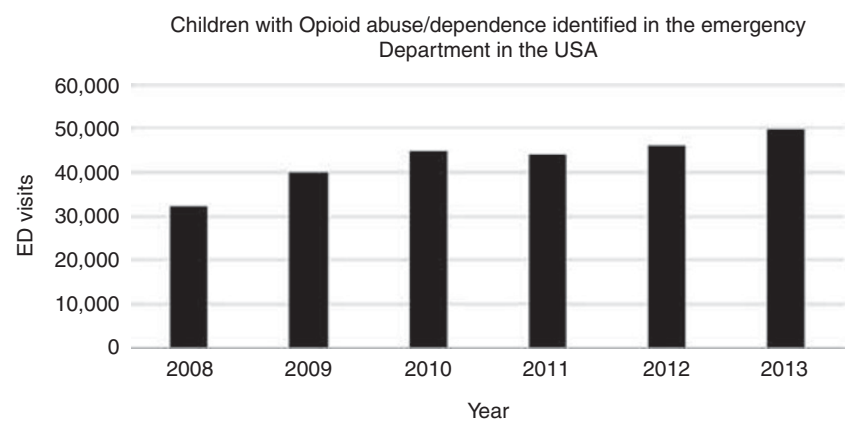

Figure 1. Trends in opioid abuse/dependence in children as identified in the Emergency Departments in the USA.

Presentations: Limited results of this study were submitted for potential presentation at AAP (American Academy of Pediatrics) 2017.

Disclosure: The authors declare no conflict of interest.

\footnotetext{
Veerajalandhar Allareddy ${ }^{1}$, Sankeerth Rampa ${ }^{2}$ and Veerasathpurush Allareddy ${ }^{3}$

${ }^{1}$ Division of Critical Care, Department of Pediatrics, Stead Family Children's Hospital, University of Iowa, Iowa City, Iowa;
}

${ }^{2}$ College of Public Health, University of Nebraska Medical Center, Omaha, Nebraska; ${ }^{3}$ College of Dentistry, University of Iowa, Iowa City, Iowa.

\section{Correspondence: Veerajalandhar Allareddy \\ (veerajalandhar-allareddy@uiowa.edu)}

\section{AUTHOR CONTRIBUTIONS}

All the authors conceived and/or designed the work that led to the submission, acquired data, and/or played an important role in interpreting the results; drafted and/or revised the manuscript; and approved the final version.

\section{REFERENCES}

1. The Opioid Epidemic: by the Numbers (https://www.hhs.gov/sites/default/ files/Factsheet-opioids-061516.pdf) Accessed 16 April 2017.

2. Drug Overdose Deaths in the United States Continue to Increase in 2015 (https://www.cdc.gov/drugoverdose/epidemic/) Accessed 16 April 2017.

3. Gaither JR, Leventhal JM, Ryan SA, Camenga DR. National trends in hospitalizations for opioid poisonings among children and adolescents, 1997 to 2012. JAMA Pediatr 2016;170:1195-201.

4. Tadros A, Layman SM, Davis SM, Bozeman R, Davidov DM. Emergency department visits by pediatric patients for poisoning by prescription opioids. Am J Drug Alcohol Abuse. 2016;42:550-5.

5. The Nationwide Emergency Department Sample (NEDS) is a Set of Hospital-Based Emergency Department Databases Included in the HCUP Family. NEDS Overview, Agency for Health Care Research and Quality (https://www.hcup-us.ahrq.gov/nedsoverview.jsp). 